\title{
Label-Free Protein Recognition 2D Array Using Nanomechanical Sensors
}

\author{
Min Yue ${ }^{1,2}$, Jeanne C. Stachowiak ${ }^{1}$, Henry Lin $^{3}$, Ram Datar $^{3,4}$, Richard Cote $^{3}$, Arun \\ Majumdar ${ }^{1,5, *}$ \\ ${ }^{1}$ Department of Mechanical Engineering, University of California, Berkeley, CA 94720. \\ ${ }^{2}$ Presently at Applied Biosystems, Foster City, CA 94404. \\ ${ }^{3}$ Department of Pathology, University of Southern California, Los Angeles, CA 90089. \\ ${ }^{4}$ Presently at Oak Ridge National Laboratory, Oak Ridge, TN 37831 \\ ${ }^{5}$ Materials Sciences Division, Lawrence Berkeley National Laboratory, Berkeley, CA
} 94720.

SUPPORTING INFORMATION: Materials and methods of immobilization of capture molecules and passivation.

Parallel Detection. Each cantilever was constructed with a reflection pad at its free end (Figure 1C). The thin arm of the cantilever was curved due to the residue stress in and between the gold and the silicon nitride layers. However, a closed square structural ridge on the reflection pad kept the pad relatively flat because it produced a high moment of inertia in the pad region ${ }^{12}$. When a collimated light beam illuminates a cantilever 
array, the curvature of each cantilever diverges the reflection from the thin arm so that a CCD camera can collect only the reflection from the flat reflection pads ${ }^{13}$. If a cantilever deflects, the direction of the reflected light will change. Therefore, the reflection spot on the CCD camera will move correspondingly. Since the CCD camera tracks and records the movement of all the spots at the same time, the deflections of multiple cantilevers in the array is monitored simultaneously.

Passivation. Bovine serum albumin (BSA) (Pierce, IL) and 2[Methoxypoly(ethyleneoxy)-propyl]thimethoxysilane (PEG-silane) (Gelest, PA) were used to passivate the silicon-nitride surface of the cantilevers and inner surfaces of the fluidic chambers. In the method of PEG-silane passivation, immediately before the experiments, the microcantilever chips were first rinsed thoroughly with isopropanol, ethanol and toluene, followed by an immersion in a freshly-prepared solution of PEGsilane in toluene for 1 hour. To make such PEG-silane solution, $100 \mu \mathrm{l}$ of PEG-silane and $40 \mu \mathrm{l}$ of concentrated $\mathrm{HCl}$ were mixed with $50 \mathrm{ml}$ of toluene in a freshly-cleaned glass beaker and the mixture was sonicated for 10 minutes. After immersion in the PEG-silane solution, the chips were rinsed with toluene, ethanol and de-ionized (DI) water to remove non-grafted PEG-silane. The chips soaked in DI water were then ready for the functionalization.

Alternatively, we also used bovine serum albumin (BSA) (Pierce, IL) to passivate the silicon nitride surface of the cantilevers and the fluidic chamber inner-wall. The chips were immersed in a solution of $2 \mathrm{mg} / \mathrm{ml}$ of BSA in phosphate buffered saline (PBS) solution overnight at $4^{\circ} \mathrm{C}$. The chips were then rinsed thoroughly with DI water and were ready for the subsequent surface functionalization. 
Surface Functionalization. We explored three methods for the immobilization of antibodies on the gold surfaces. The first immobilization method utilized 3,3'-Dithiobis[sulfosuccinimidylpropionate] (Pierce, IL), a.k.a. DTSSP, to directly crosslink the antibody to the gold surface. The cantilevers were immersed in freshly prepared $2 \mathrm{mM}$ DTSSP in citrate buffer (5mM, pH 5) for 2 hours. The weak acidic citrate buffer prevented the NHS esters from being hydrolyzed while the Au-S bonds formed. The wells were then rinsed with citrate buffer to remove the excessive DTSSP in solution. Immediately after washing, $100 \mu \mathrm{g} / \mathrm{ml}$ monoclonal anti-PSA antibody in PBS (phosphate buffer saline, $\mathrm{pH}=7.4$ ) was injected into the reaction chambers to allow for antibody coupling via DTSSP. The cantilevers were incubated in the antibody solution for at least 5 hours to allow for maximum immobilization of the antibody onto the gold surface, which occurred through covalent bonding between the amine groups of the antibody and the N-hydroxysulfosuccinimide (sulfo-NHS) ester of DTSSP.

The second method of surface functionalization was to form a mixed SAM by using a mixture of NHS (N-hydroxysuccinimide) thiol (HS-[CH 2$] 11-\left[\mathrm{OCH}_{2}\right]_{6}-\mathrm{COO}-\mathrm{NHS}$ ) (NHS-thiol) and HS-[CH$]_{11}-\left[\mathrm{OCH}_{2}\right]_{6}-\mathrm{OH}$ (PEG-thiol), obtained from Prochimia, Poland. NHS-thiol and PEG-thiol were dissolved in pure ethanol and were mixed to a final concentration of $3.2 \mathrm{mM}$ and $0.8 \mathrm{mM}$, respectively. The cantilevers were immersed in the mixture for 2 hours for surface modification, followed by an ethanol rinse and a citrate buffer rinse. Immediately after rinsing, the cantilevers were incubated in the antibody solution (100 $\mu \mathrm{g} / \mathrm{ml})$, similar to the DTSSP protocol. The rest of the process was the same as in the DTSSP method. 
The third means of attaching antibody molecules to gold surfaces was to utilize the strong binding affinity between biotin and NeutrAvidin ${ }^{\mathrm{TM}}$. We used EZ-Link Sulfo-NHSSS-Biotin (Sulfosuccinimidyl 2-(biotinamido)-ethyl-1,3-dithiopropionate) and NeutrAvidin ${ }^{\mathrm{TM}}$ Biotin-Binding Protein (Pierce, IL). The cantilevers were immersed in 2mM Sulfo-NHS-SS-Biotin solution for 2 hours, allowing for grafting of Sulfo-NHS-SSBiotin to the gold surface of the cantilevers by the Au-S bond. After washing with PBS, the cantilevers were exposed to the NeutrAvidin ${ }^{\mathrm{TM}}$ solution of $100 \mu \mathrm{g} / \mathrm{ml}$ for 4 hours. The NeutrAvidin ${ }^{\mathrm{TM}}$ easily conjugated to the Sulfo-NHS-SS-Biotin on the gold surface due to the strong affinity between biotin and NeutrAvidin ${ }^{\mathrm{TM}}\left(K_{a}=10^{15} \mathrm{M}^{-1}\right)$. Using the biotinylation kit (Pierce Inc. USA), the anti-PSA antibody was biotinylated for the subsequent coupling through NeutrAvidin ${ }^{\mathrm{TM}}$. The pre-coated cantilevers were immersed in the $100 \mu \mathrm{g} / \mathrm{ml}$ biotinylated antibody solution for 4 hours to achieve attachment between the biotinylated antibody and the NeutrAvidin ${ }^{\mathrm{TM}}$ on the gold surface. The chip was kept at $4{ }^{\circ} \mathrm{C}$ to preserve the proteins in all steps of each of the three functionalization protocols. Following each of the functionalization protocols, the chips were thoroughly washed in PBS and were ready for detecting target molecules. 